\title{
An uneventful pregnancy and delivery in a metastatic adult wilms' tumor-a rare case report
}

\begin{abstract}
Adult Wilms' tumor (AWT) is a malignant embryonic renal neoplasm. It has an incidence of less than 0.2 per million per year which signifies its rarity in adults. Herein, we report a case of adult Wilms tumor who has had a successful pregnancy and delivery against her odds of a metastatic disease. There is a dearth of information about this rare disease, which makes its management quite challenging.
\end{abstract}

\author{
Volume 7 Issue 4 - 2017 \\ VijayasarathyK,' P Jovita M Martin, ${ }^{2}$ Durai \\ Mavalavan \\ 'Professor Medical Oncology, Omandarur Multispecialty \\ Hospital, India \\ ${ }^{2}$ Assistant Professor Medical Oncology, Tamil Nadu Government \\ Multisuperspeciality Hospital, India \\ ${ }^{3}$ Assistant Professor Medical Oncology, Omandarur \\ Multispecialty Hospital, India
}

Correspondence: P Jovita M Martin, Assistant Professor

Medical Oncology, Tamil Nadu Government Multisuperspeciality

Hospital Chennai, India, Email jovimartin4@gmail.com

Received: October 04, 2017 | Published: October 24, 2017

\section{Introduction}

Adult Wilms' Tumor (AWT) originates from metanephric blastema. There are over 200 cases reported in the literature cut there has no clinical trials conducted on this subgroup of patients. ${ }^{2}$ Hence, there are no protocols or consensus on the definitive multimodality management strategies designed for the management of adult Wilms tumor yet. In this scenario, it seemed worthwhile to present this case of metastatic Wilms tumor, going through a successful pregnancy and delivery.

\section{Case report}

This is a case report on a 24year old lady, a known case of Wilms tumor, diagnosed in 2009 when she was 19years of age. She was diagnosed to have left kidney Wilms tumor, biopsy showed blastemal components, monophasic, Stage III in December 2009 at Government general hospital. She had a left radical nephrectomy, splenectomy and inferior vena cava thrombectomy. The histopathology showed one of the 5 lymph nodes positive for metastatic deposits. She was given 2 cycles chemotherapy with Vincristine, Cyclophosphamide and Methotrexate followed by 6 cycles of Cisplatin, Etoposide, Cyclophosphamide and Vincristine. She had 2 more cycles of Vincristine, Cyclophosphamide and Methotrexate completed in July 2010. As she was on follow up, she was found to have lung, and bone secondaries. She was treated with palliative emergency radiation to the spine 30Gy@3Gy in 10 fractions in June 2011. Due to the progressive disease she was planned on SIOP protocol. She received one cycle of Cisplatin, Adriamycin, Cyclophosphamide and Etoposide followed by 6cycles of Ifosphamide and Adriamycin. She was given 2nd line salvage chemotherapy with ICE regimen; Ifosphamide 2gm, Etoposide 150mg and Carboplatin 450mg in January 2012. She was given oral Cyclophosphamide since February 2012 to June 2012. 6months she did not take any oral chemotherapy, in January 2013 she conceived, on October 8th 2013 she delivered a full term female baby weighing $2.75 \mathrm{Kg}$ via caesarean section. In Jan 2014, her PET CT showed increased metabolic activity in the left renal fossa 35.1x 34.7x22.6mm; bilateral level Ib and II neck LN (all LN less than $1 \mathrm{~cm})$; mediastinal and hilar nodes, ill-defined opacity in the right lung involving the upper lobe posterior segment $25.3 \times 19.2 \mathrm{~mm}$, mediastinal lymph nodes. There were irregular lytic lesions in the posterior aspect of D9 and D10. She has been on the fourth line of salvage chemotherapy.

\section{Discussion}

Nephroblastoma or Wilms' tumor accounts for around about 5-6\% of neoplasms in childhood, it occurrence is rare accounting to less than $3 \%$ in the adult population. ${ }^{3}$ The worse biological behavioural pattern of Wilms' tumor is unexplained. ${ }^{4}$ Due to the rarity of its occurrence conducting randomized trials in AWT is farfetched. Hence, this disease is often managed with the extrapolated study results of the paediatric protocols. National Wilms' Tumor Study (NWTS), SIOP and other studies have suggested multimodal therapy for the disease with surgery as the mainstay, chemotherapy is with (actinomycin D, vincristine and doxorubicin) for 15 months and tumor bed irradiation in the case of stage 3 disease. Less aggressive therapy with two drugs is advised in stage 1 and 2 disease. If the primary tumor is inoperable upfront, pre op chemotherapy followed by a second look laparotomy is opted. ${ }^{5}$ On combined modality treatment the overall survival is more than $80 \% .^{4}$ Multimodal treatment according to pediatric protocols does yield good survival rates. ${ }^{2}$ Kilton et al., ${ }^{6}$ in 1980, laid down criteria for the diagnosis of AWT. (i) The presence of a primary renal neoplasm with primitive blastematous spindle or round cell component and the formation of abortive or embryonal tubular or glomeruloid structures are necessary. (ii) There should be no area of the tumor should show features suggestive of renal cell carcinoma; (iii) The pictorial confirmation of histology is mandatory; (iv) age has to be $>15$ years. Solid malignancies in young fertile adults are quite commonly encountered in Oncology. Issue of child bearing and its related emotional turmoil in these young adults cannot be denied and are not uncommon. This case is reported for its uniqueness, a metastatic AWT patient, having gone through a successful pregnancy. Despite the stage and the prognosis, this patient has gone through 
a full term pregnancy delivering a normal healthy girl baby. She is stilling battling the disease as she has been planned on the fourth line of salvage chemotherapy.

\section{Conclusion}

Malignancies like adult Wilms tumor, due to its rarity do not have a proper consensus for treatment. However the literature review has given us evidence of good prognosis when the paediatric protocols are extrapolated on the adult Wilms. The current case report helps us to challenge the nature of the disease for a normal pregnancy in a metastatic solid tumor such as AWT.

\section{Acknowledgements}

None.

\section{Conflict of interest}

The author declares no conflict of interest.

\section{References}

1. Mitry E, Ciccolallo L, Coleman MP, et al. Incidence of and survival from Wilms' tumour in adults in Europe: data from the EUROCARE study. Eur J Cancer. 2006;42(14):2363-2368.

2. Mriganka Sharma. Adults Wilms Tumor. Saudia Arabia journal of Kidney diseases and transplantation. 2009;20(5):835-837.

3. Hentrich MU, Brack NG, Schmid P, et al. Harten- stein RC: Adult Wilms' tumor-Report of two cases and review of literature. Cancer. 1995;75(2):545-551.

4. Reinhard Harald, Aliani Schahin, Ruebe Christian, et al. Wilms' tumor in adults: results of the society of pediatric oncology (SIOP) 93-01/society for pediatric oncology and hematology (GPOH) study. Journal of Clinical Oncology. 2004;22:4500-4506.

5. Grant Williams RA, Colbeck NF. Growing adult Wilms' tumour: Review of 14patients. Br J Urol. 1992;70:230-235.

6. Kilton L, Mathews MJ, Cohen MH. Adult Wilms' tumour: a report of prolonged survival and review of literature. J Urol. 1980;124(1):1-5. 